Check for updates

Cite this: J. Mater. Chem. A, 2018, 6, 19974

Received 19th July 2018

Accepted 18th September 2018

DOI: $10.1039 / c 8 t a 06983 b$

rsc.li/materials-a

\section{Silicon monophosphide as a possible lithium battery anode material $\uparrow$}

\author{
R. Reinhold, (D) ${ }^{* a b}$ D. Mikhailova, (D) a T. Gemming, (D) ${ }^{a}$ A. B. Missyul, (D) ${ }^{c}$ C. Nowka, ${ }^{a}$ \\ S. Kaskel (D) ${ }^{b}$ and L. Giebeler (D)*a
}

Binary (semi)metal-phosphorus compounds fascinate nowadays as they promise high specific capacities in metal ion batteries and an advantageous in situ formation of the electrochemically active material inside the electrochemical cell without air exposure. Here, we report on SiP as a new member of this material family which was tested as an anode material in Li ion batteries due to a highly attractive theoretical specific capacity of about $3000 \mathrm{mAh} \mathrm{g}^{-1} \mathrm{SiP}$. After synthesis by a vapour-transport reaction a cotton wool-like product was obtained revealing a layered $2 \mathrm{D}$ crystalline microribbon-like morphology, space group $\mathrm{Cmc2}_{1}$, which may allow fast Li ion intercalation/diffusion kinetics. SiP also opens up a novel perspective for application in ultra-thin optoelectronics or flexible photovoltaics. During the first discharging half-cycle the crystalline phase amorphizes as indicated by operando synchrotron powder diffraction followed by an amorphous state without re-formation of any crystalline phase, including $\mathrm{Li}_{3} \mathrm{P}$. Our investigations after 50 dischargingcharging cycles show a specific capacity of $550 \mathrm{mAh} \mathrm{g}^{-1}$, which supports the trend of lifetime reduction by LiP formation. Further optimisation is necessary to allow a broad application for novel technologies like high performance $\mathrm{Li}$ ion or $\mathrm{Li}-\mathrm{S}$ batteries.

Lithium ion batteries have revolutionized the mobile devices market starting in the early 1990s and are just about to do the same in the automotive and even in the stationary storage sectors. To allow for higher efficiency batteries with much increased electrical energy storage capacities, many efforts have focused on introducing high power, high performance

${ }^{a}$ Leibniz Institute for Solid State and Materials Research (IFW) Dresden e.V., Helmholtzstraße 20,D-01069 Dresden, Germany.E-mail: l.giebeler@ifw-dresden.de; r.reinhold@ifw-dresden.de

${ }^{b}$ Department of Inorganic Chemistry, Technische Universität Dresden, Bergstraße 66, D-01069 Dresden, Germany

${ }^{c}$ CELLS-ALBA Synchrotron, Carrer de la Llum 2-26, 08290 Cerdanyoladel Vallèes, Barcelona, Spain

$\dagger$ Electronic supplementary information (ESI) available: Crystal structure, HRTEM, STEM, STEM-EDXS, differential capacity plot. See DOI: $10.1039 / \mathrm{c} 8 \mathrm{ta} 06983 \mathrm{~b}$ materials, e.g. to achieve customers' range demands for e-vehicles. Here, the classical graphite negative electrode reaches its limit when much higher lithium uptake is necessary as typical for $\mathrm{Li}-\mathrm{S}$ batteries. Silicon compounds represent highly promising candidates to replace graphite as the anode material as already partially realized in commercial battery technology.$^{1-4}$ However, volume expansion and consequently pulverization ${ }^{5}$ of the material is still the greatest obstacle to solve which, in consequence, leads to conductivity losses in the electrode composite and the repeated formation of new surfaces supporting parasitic electrolyte consumption. ${ }^{6}$ Many studies therefore focused on the design of nano-silicon morphologies such as nanowires, ${ }^{7-10}$ nanoparticles ${ }^{11,12}$ or nanopillars. ${ }^{13,14}$ Nanoparticles below $20 \mathrm{~nm}$ also serve as an easy-to-reach alternative to the high-tech preparation of nanoarchitectures and show similar performance in metal ion batteries. An intelligent gimmick in the sense of nanoparticle synthesis is the application of transition metal phosphides ( $\mathrm{MP}, \mathrm{M}=\mathrm{Cu}, \mathrm{Co}$, $\mathrm{Sn}) .{ }^{15-19}$ By a complete conversion $\mathrm{Li}_{3} \mathrm{P}$ and active metallic nanoparticles are formed in situ.

In particular, studies regarding $\mathrm{Sn}_{4} \mathrm{P}_{3}$ show a broad applicability in lithium, ${ }^{17,20}$ sodium $^{21-23}$ and potassium ${ }^{24,25}$ ion batteries. Due to the similar chemical properties, the electrochemical behaviour of binary Si-P compounds should be similar. However, this work focuses on lithium-based batteries as silicon shows a high ability to form intermetallic phases with lithium compared to sodium or potassium. To reach high specific capacities low elemental weights are recommended and can be realized by combining silicon and phosphorus. Si-Pcompounds represent a new class of future anode materials where silicon diphosphide $\left(\mathrm{SiP}_{2}\right)$ has already attracted high attention due to its high theoretical capacity of $2900 \mathrm{mAh} \mathrm{g}^{-1}$ $\left(\mathrm{Li}_{15} \mathrm{Si}_{4}, \mathrm{Li}_{3} \mathrm{P}\right) .{ }^{26-28}$

The high abundance of silicon and phosphorus combined with their electrochemical mechanism of alloying and conversion mechanism justifies a scientific effort to implement the material into metal ion batteries. A relatively fast deactivation of $\mathrm{SiP}_{2}$ by successive and irreversible LiP shell formation 
establishes an insulating layer around the Si particle core. ${ }^{28}$ Reducing the phosphorus content, as in the case of silicon monophosphide, benefits an even higher theoretical specific capacity (3060 mAh g ${ }^{-1} ; \mathrm{Li}_{15} \mathrm{Si}_{4}, \mathrm{Li}_{3} \mathrm{P}$ ) compared to $\mathrm{SiP}_{2}$ and may avoid excessive LiP formation. Moreover, $\mathrm{SiP}$ is expected to form in situ electrochemically active silicon nanoparticles at reduced volume changes. Addressing the latter two scenarios simultaneously, the lifetime of the battery may be significantly increased, especially compared to $\mathrm{SiP}_{2} \cdot{ }^{28}$ An interesting feature of the used synthesis is found in the self-organization of SiP in a layered 2D ribbon-like material in a bottom-up approach without additional handling and forms Si nanoparticles in situ during the electrochemical reaction, e.g. without further temperature treatment and no complex deposition methods are applied as needed for other nano-sized silicon morphologies. ${ }^{7-10,13,14}$

From the Si-P binary phase diagram developed by Olesinski, ${ }^{29}$ silicon monophosphide is the only stable phase under ambient pressure conditions, emphasizing its thermodynamic stability compared to $\mathrm{SiP}_{2}$. Regarding silicon monophosphide, only a few synthesis procedures have been described in combination with crystal structure studies. First syntheses were realized in the 1960s via vapour-growth reactions ${ }^{30,31}$ and recently by a high pressure-high temperature synthesis in a cubic multi-anvil cell ${ }^{32}$ leading to bulk crystal materials for both techniques. This group IV-V monopnictide SiP shows an orthorhombic (Cmc2 1 space group) 2D layered structure, where single layers are stacked by weak van der Waals interactions. ${ }^{33}$ First DFT calculations ${ }^{33}$ demonstrated comparable exfoliation energies as for graphite which enable exfoliation by mechanical stress resulting in semiconducting SiP layers as an interesting candidate for applications in thin, bendable photovoltaics and optoelectronics. ${ }^{33}$

We report on the optimized SiP synthesis via a chemical vapour-transport reaction obtaining layered $2 \mathrm{D}$ materials and give for the first time deeper insights into the structural properties and electrochemical behaviour. This work mainly focuses on the electrochemical properties of SiP as a negative electrode material for lithium ion batteries. Another key point considers the structural changes during lithiation to get an idea on a possible structure preservation which may be reachable with the observed layered arrangement as would be untypical for phosphide compounds when treated under similar electrochemical conditions where they undergo a conversion reaction to the elemental state and usually $\mathrm{Li}_{3} \mathrm{P}$.

Our results reveal moderate electrochemical activity and are still under improvement to enhance the specific capacity and lifetime.

After synthesis with an exothermic chemical vapour-transport reaction $\left(T_{1} \rightarrow T_{2}, T_{2}>T_{1}\right)$, a cotton wool-like product was obtained at $T_{2}=1000{ }^{\circ} \mathrm{C}$ revealing a layered $2 \mathrm{D}$ microribbonshaped morphology as photographed by SEM (Fig. 1) where the macroscopically visible, exfoliatable layers are marked with arrows in the inset. The corresponding EDXS mappings, displayed in Fig. $\mathrm{S}-1, \uparrow$ confirm the homogeneous distribution of $\mathrm{Si}$ and $\mathrm{P}$ throughout the sample.

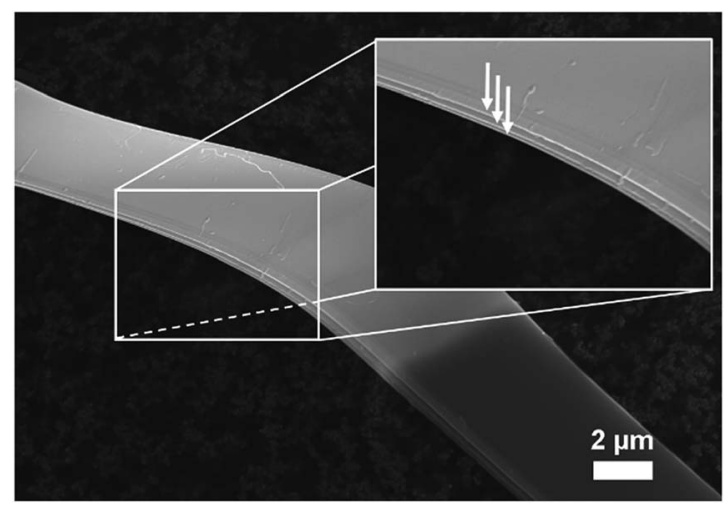

Fig. 1 SEM image of as-prepared SiP. Macroscopic exfoliatable layers are indicated by arrows in the inset.

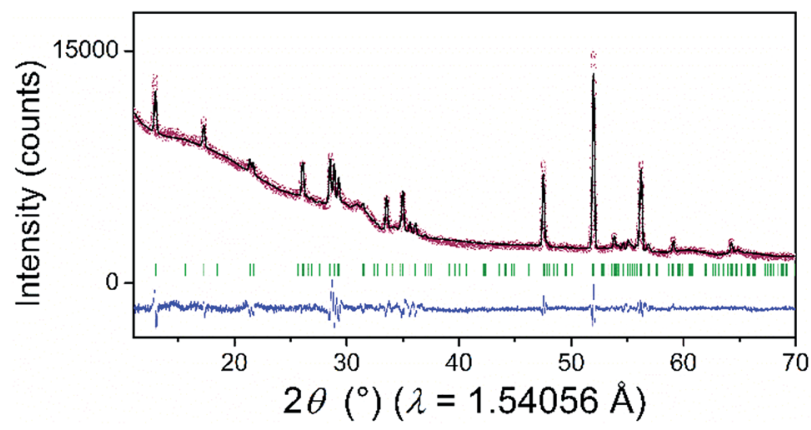

Fig. 2 XRD pattern of the as-prepared SiP (red dots) with the calculated profile (black solid line) according to the Le Bail analysis of the structural model from SiP. The difference curve resulted from the subtraction of the observed from the calculated data (blue solid line). Green vertical lines represent the Bragg positions of the reflections according to the SiP-space group $\mathrm{Cmc2}_{1}$-structural model known from the literature. ${ }^{30,31}$

The powder XRD pattern of the as-synthesized SiP (Fig. 2) is in good agreement with literature data ${ }^{30,31}$ and is analysed with the corresponding SiP structure model with orthorhombic space group $C m c 2_{1}$ as shown in Fig. S-2. $\uparrow$ SiP lattice parameters were determined by a Le Bail fit to $a=3.51473(7) \AA, b=$ 20.4880(5) $\AA$, $c=13.6198(4) \AA$. The structure is described as layered in the (001) direction. Due to the weak van der Waals bonds between the layers, SiP may be favourable for ion intercalation as well as exfoliation of individual layers. Selected area electron diffraction (SAED) (Fig. 3) shows that a ribbon of asprepared SiP is characterized by a single crystalline habit due to the absence of additional, extrinsic diffraction spots or diversely oriented individuals like typical for twins. ${ }^{31}$

Additionally, high resolution transmission electron microscopy (HRTEM) images (Fig. S-3 and S-4†) nicely present the homogeneous arrangement of atoms within the layers. STEMEDXS measurements (Fig. S-5†) were recorded to verify the elemental composition. The spectra perfectly support the expected content of Si-to-P ratio of $1: 1$ and show no signal for iodine (transporting agent). As previously reported, ${ }^{28} \mathrm{SiP}_{2}$ reacts during lithiation in a three-step mechanism: (1) a conversion reaction to intermediate $\operatorname{LiP}_{x}$ phases $(1 \leq x \leq 7)$ followed by (2) 


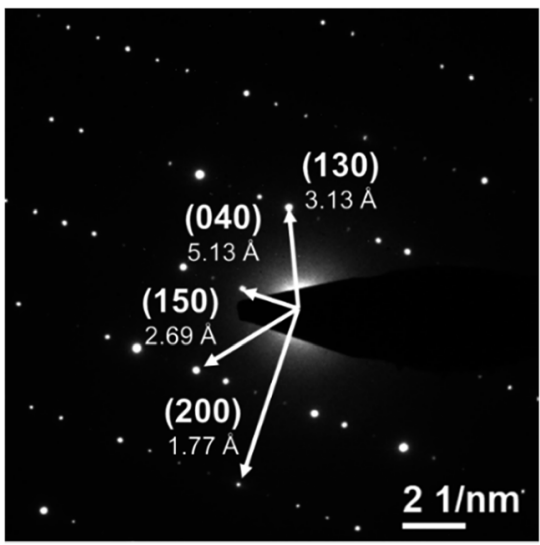

Fig. 3 SAED pattern of as-prepared SiP with indexed diffraction spots along the (001) zone axis.

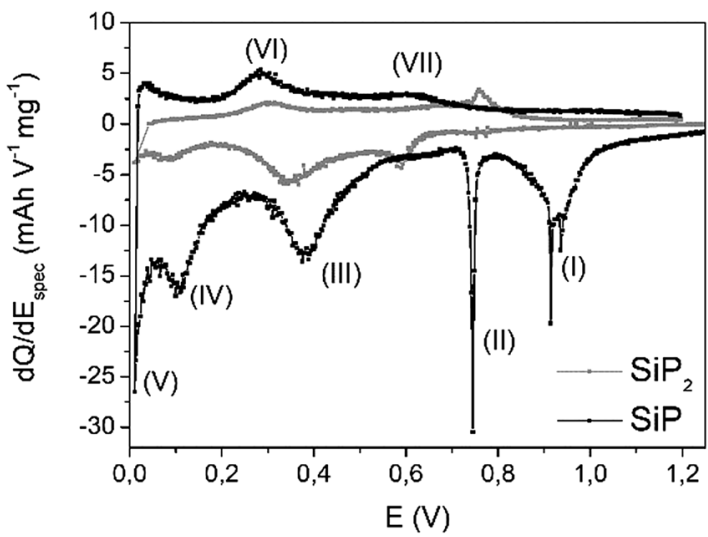

Fig. 4 Differential capacity plot (DCP) of SiP (black) during the first cycle. For comparison, DCP of $\mathrm{SiP}_{2}$ (grey) is given as a reference.

the formation of $\mathrm{Li}_{3} \mathrm{P}$, and, finally, (3) the alloying of $\mathrm{Li}$ and $\mathrm{Si}$ to $\mathrm{Li}_{x} \mathrm{Si}$. In comparison to $\mathrm{SiP}_{2}$, the differential capacity plot (DCP) of SiP during the first cycle, displayed in Fig. 4, shows similar redox behaviour. For further discussion, discharging is defined as insertion of Li ions while charging describes the removal. For better illustration, the first derivative of the current is calculated normalized to the mass of SiP.

Comparing the potential window of $0.8-1.0 \mathrm{~V} v s$. $\mathrm{Li} / \mathrm{Li}^{+}$, various redox peaks $(\mathrm{I}+\mathrm{II})$ occur, which most likely indicate structural changes due to the sharp signals. This is closely associated with a relatively low kinetic barrier. Based on the absence of both signals (Fig. S-6†) in the second cycle, irreversible processes like the formation of a solid electrolyte interphase (SEI) or the lithiation of a carbon additive at $0.74 \mathrm{~V}$ might be possible. ${ }^{34}$

The reaction to $\mathrm{Li}_{3} \mathrm{P}$ (III) proceeds around $0.4 \mathrm{~V}$ and nicely fits the previous results of $\operatorname{SiP}_{2}{ }^{28}$ Below $0.1 \mathrm{~V}$, alloying of silicon nanoparticles (IV, V) should occur. During delithiation, reaction (IV) at $320 \mathrm{mV}$ may correspond to the dealloying of $\mathrm{Li}_{x} \mathrm{Si}$ particles, which is also observed for pure silicon in the same range. ${ }^{11,35}$ At about $0.6 \mathrm{~V}$ vs. $\mathrm{Li} / \mathrm{Li}^{+}$during $\mathrm{Li}$ removal, the decomposition of $\mathrm{Li}_{3} \mathrm{P}$ species proceeds. Interestingly, all lithiation processes are slightly shifted to higher voltages whereas the corresponding delithiation reactions are found at somewhat lower voltages leading to the assumption of faster kinetics. Comparing the currents of both redox processes, a higher current is observed during the lithiation process implying a low coulombic efficiency in the first cycle.

For further insights on possible changes, e.g. structural transitions, phase formations, dependent on the scanning voltage, operando synchrotron XRD was performed and the results are displayed in Fig. 5.

For better clarity, only the diffraction patterns between $12^{\circ}$ and $15^{\circ} 2 \theta$ are presented. The intensity of the reflections of the crystalline SiP (indexed with red dashed lines) decreases continuously and vanishes at voltages below $0.1 \mathrm{~V} v s$. $\mathrm{Li}^{-} \mathrm{Li}^{+}$. During Li insertion into the active material, no shift of the reflections to higher or lower angles is observed. According to these results, an intercalation of $\mathrm{Li}$ ions in-between the SiP layers like for graphite is excluded. Moreover, in the fully lithiated state, no reflections of crystalline $\mathrm{Li}_{3} \mathrm{P}$ appear, which is in contrast to our previous study regarding $\mathrm{SiP}_{2}$. Three reasons can explain this behaviour. (1) The Li intercalation mechanism in the case of SiP differs from the one of $\mathrm{SiP}_{2}$. (2) $\mathrm{Li}_{3} \mathrm{P}$ is formed but the critical size of the particles to induce crystallization has not been reached in the first discharging cycle. $\mathrm{Li}_{3} \mathrm{P}$ therewith may exist in an amorphous state. (3) Phosphorus only reacts to LiP which, however, is amorphous as reported previously for $\mathrm{SiP}_{2} \cdot{ }^{28}$ For further analysis of the electrochemical performance, galvanostatic cycling of $\mathrm{SiP}$ electrodes in a two-electrode configuration $v s$. $\mathrm{Li}^{-\mathrm{Li}^{+}}$was conducted. The $\mathrm{SiP}$ cells were cycled with a current density of $100 \mathrm{~mA} \mathrm{~g}{ }^{-1}$, which corresponds to a C-rate of about $\mathrm{C} / 30$.

In the first charging cycle, capacities of $1000 \mathrm{mAh} \mathrm{g}^{-1}$ (Fig. 6) are retained which highlights the potential of this anode material. As expected from DCP during the first cycle, low coulombic efficiencies of $32 \%$ are observed. This observation is most likely attributed to structural changes and irreversible reactions, SEI formation, etc. during cycling. The formation of an insulating LiP phase as observed for other metal phosphides might be possible but we have not found any evidence for it. Further investigations regarding intermediate phases are in progress. However, after 50 cycles, delithiation capacity remains constant at around $550 \mathrm{mAh} \mathrm{g}{ }^{-1}$ showing high desirable

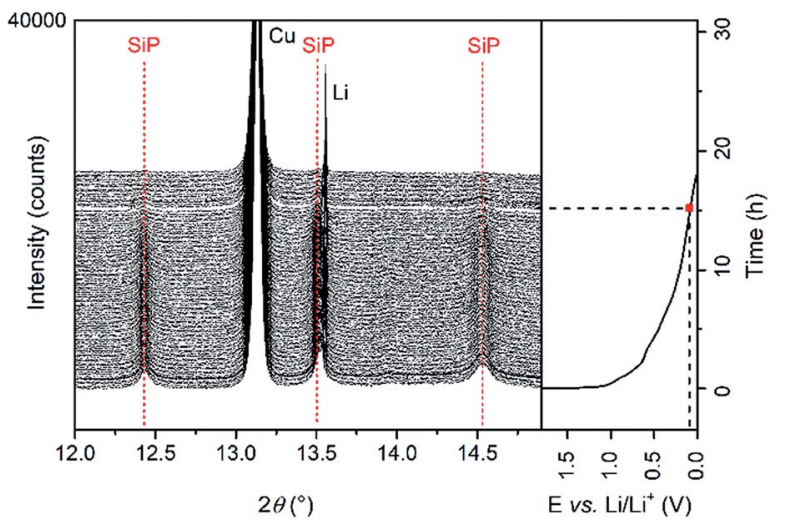

Fig. 5 Operando synchrotron XRD pattern collected during the first discharging cycle. Red dashed lines indicate vanishing reflections. 


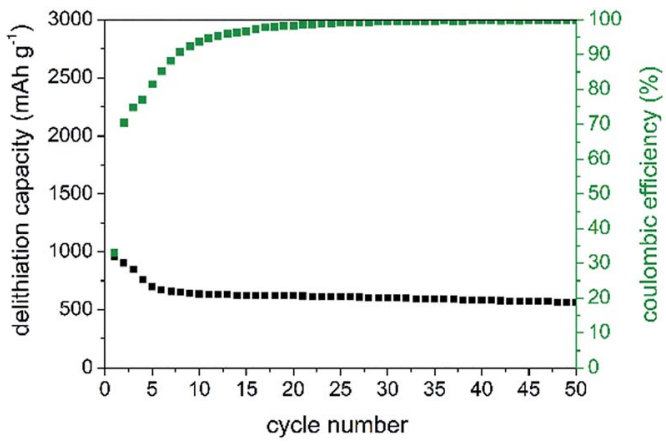

Fig. 6 Delithiation capacity and corresponding coulombic efficiencies (green) of SiP

coulombic efficiencies of 99.6\%. Additionally, SiP offers a good rate capability at high current densities of up to $500 \mathrm{~mA} \mathrm{~g}^{-1}$, as displayed in Fig. S-7.†

\section{Conclusions}

We herein reported an optimized synthesis of SiP via a vapourtransport reaction using iodine as the transport agent. As a product, microribbon-like $\mathrm{SiP}$ with an orthorhombic $\mathrm{Cmc2}_{1}$ space group is obtained. TEM measurements combined with XRD confirm the crystal structure and elemental ratio of $1: 1$ (Si : P). First electrochemical studies highlighted its high potential as an anode material since a capacity of $1000 \mathrm{mAh} \mathrm{g}^{-1}$ after the first discharging cycle was demonstrated. However, the material suffers from fast capacity fading due to an obviously incomplete reaction. Therefore, further conceptual development is required to avoid the LiP insulating shell formation.

\section{Experimental section}

\section{Synthesis of SiP}

Silicon monophosphide (SiP) was synthesized via a transport reaction of pure silicon (Aldrich, -325 mesh, 99\% purity) and red phosphorus (Alfa Aesar, -100 mesh, $98.9 \%$ purity) powders. Iodine (Merck, 99\%) was added as a transporting agent. $50 \mathrm{mg}$ of a stoichiometric mixture of silicon and phosphorus and $6 \mathrm{mg}$ of iodine (placed in an extra tube) were filled in a quartz ampoule. The ampoule was sealed under vacuum $\left(10^{-3} \mathrm{mbar}\right)$ and was placed into a horizontal two-zone furnace. In a first equilibrating step, the temperature was set to $400{ }^{\circ} \mathrm{C}$ for $16 \mathrm{~h}$ to guarantee an equilibrium state of phosphorus partial pressure. In a second step, the temperature was increased to $900{ }^{\circ} \mathrm{C}\left(T_{1}\right.$, source $)$ and $1000{ }^{\circ} \mathrm{C}\left(T_{2}\right.$, sink $)$ and kept for $160 \mathrm{~h}$ to allow the exothermic reaction to proceed. This temperature gradient was chosen based on the studies on other metalphosphides. ${ }^{36}$ Finally, the ampoule was cooled down naturally. All following steps to work with the SiP were conducted under an inert gas atmosphere in a glovebox.

\section{Material characterization}

X-ray diffraction (XRD) patterns were recorded on a Stadi $\mathrm{P}$ diffractometer (STOE) with a curved Ge(111)-crystal as the monochromator. Samples were filled in a glass capillary
(Hilgenberg, Nr. 10, diameter $0.7 \mathrm{~mm}$ ) and fire-sealed. $\mathrm{Cu} \mathrm{K}_{\alpha 1}$ radiation $(\lambda=1.54056 \AA)$ was applied. Capillaries were measured in Debye-Scherrer mode using a step width of $\Delta 2 \theta=$ $0.02^{\circ}$. FullProf implemented into the software WinPlotR was used for performing Le Bail analyses. The patterns were analysed according to literature data for SiP. ${ }^{30}$

Operando powder synchrotron diffraction measurements on $\mathrm{SiP}$ in a coin cell with a glass window of $3 \mathrm{~mm}$ in diameter were performed at the beamline BL04-MSPD ${ }^{37}$ at ALBA (Barcelona, Spain) in a transmission mode. A Li disk served as an anode. The experimental setup containing a coin cell holder connected to a VMP multichannel potentiostat is described elsewhere. ${ }^{38}$ Data were collected every 9 minutes in steps of $0.005^{\circ}$ at $\lambda=$ $0.41310(1) \AA$, which were refined from the reflection positions of a NIST $\mathrm{LaB}_{6}$ reference material.

In order to characterize pristine $\mathrm{SiP}$, a pattern was recorded before starting the electrochemical process. The cell was then successively discharged in galvanostatic mode at a constant current of $100 \mathrm{~mA} \mathrm{~g}^{-1}$. The $\mathrm{Cu}$ mesh current collector on the positive electrode side was used as an internal standard during the measurements.

Scanning electron microscopy (SEM) was performed on a Gemini LEO 1530 (Zeiss) with an acceleration voltage of $10 \mathrm{kV}$.

Transmission electron microscopy (TEM) experiments were carried out on a FEI Tecnai F30 with $300 \mathrm{kV}$ acceleration voltage equipped with a field emission gun. The material was dispersed in dimethyl carbonate (DMC) through sonication and drop coated onto a copper grid with a lacey carbon layer as the sample holder. Selected area electron diffraction (SAED), high resolution transmission electron microscopy (HRTEM) images and scanning transmission electron microscopy energy-dispersive X-ray spectroscopy (STEM-EDX) images were taken.

\section{Electrode preparation}

For electrode preparation, $30 \mathrm{wt} \% \mathrm{SiP}$ was mixed with $60 \mathrm{wt} \%$ Super P (Timcal) and $10 \mathrm{wt} \%$ PVDF 1013 (Solvay). The mixture was dissolved in $N$-methylpyrrolidone (NMP, Sigma Aldrich), drop-coated onto a copper foil $(\varnothing 12 \mathrm{~mm})$ and dried at $80{ }^{\circ} \mathrm{C}$ overnight under vacuum. For assembling the cells in a twoelectrode configuration, a lithium metal disc (Chempur, $250 \mu \mathrm{m}$ thickness), two glass fibre separators (Whatman) and $250 \mu \mathrm{l}$ electrolyte ( $1 \mathrm{M} \mathrm{LiPF}_{6}$ in dimethyl carbonate (DMC)/ethylene carbonate (EC) $(1: 1 \mathrm{v} / \mathrm{v}), \mathrm{LP} 30, \mathrm{BASF})$ and the SiP composite as the working electrode were arranged in a Swagelok cell casing. Electrochemical tests were realized with a multichannel VMP3 potentiostat (BioLogic) at a constant temperature of $25{ }^{\circ} \mathrm{C}$. Galvanostatic cycling with potential limitation (GCPL) was conducted between 0.01 and $1.2 \mathrm{~V} v$ s. $\mathrm{Li}_{/} / \mathrm{Li}^{+}$at a current density of $100 \mathrm{~mA} \mathrm{~g}^{-1}$. For further characterization, different current densities in the range of $50-200 \mathrm{~mA} \mathrm{~g}^{-1}$ were applied. The specific capacity and current density were calculated based on the mass of silicon monophosphide.

\section{Conflicts of interest}

There are no conflicts to declare. 


\section{Acknowledgements}

We would like to thank $\mathrm{K}$. Wruck for assisting us in the synthesis of SiP. Beamtime allocation at the BL04-MSPD beamline at the ALBA Synchrotron in Barcelona, Spain, is gratefully acknowledged. This work was partially supported with funding of the German Federal Ministry of Education and Research (BMBF) in the WING center BamoSa- Battery mobility in Saxony (grant no. 03X4637) and the European Union/European Regional Development Fund (ERDF) and the Free State of Saxony in the TTKin project (SAB grant no. 100225299).

\section{Notes and references}

1 M. Jeong, H. L. Du, M. Islam, J. K. Lee, Y. Sun and H. Jung, Nano Lett., 2017, 17, 5600-5606.

2 I. H. Son, J. H. Park, S. Kwon, S. Park, M. H. Rümmeli, A. Bachmatiuk, H. J. Song, J. Ku, J. W. Choi, J. Choi, S.-G. Doo and H. Chang, Nat. Commun., 2015, 6, 7393.

3 D. Nan, Z. Huang, R. Lv, Y. Lin, L. Yang, X. Yu, L. Ye, W. Shen, H. Sun and F. Kang, J. Nanomater., 2014, 139639.

4 M. N. Obrovac and L. J. Krause, J. Electrochem. Soc., 2007, 154, A103-A108.

5 M. N. Obrovac and L. Christensen, Electrochem. Solid-State Lett., 2004, 7, A93-A96.

6 T. Jaumann, J. Balach, U. Langklotz, V. Sauchuk, M. Fritsch, A. Michaelis, V. Teltevskij, D. Mikhailova, S. Oswald, M. Klose, G. Stephani, R. Hauser, J. Eckert and L. Giebeler, Energy Storage Materials, 2017, 6, 26-35.

7 L.-F. Cui, R. Ruffo, C. K. Chan, H. Peng and Y. Cui, Nano Lett., 2009, 9, 491-495.

8 B. Laïk, L. Eude, J. P. Pereira-Ramos, C. S. Cojocaru, D. Pribat and E. Rouvière, Electrochim. Acta, 2008, 53, 5528-5532.

9 A. Krause, S. Dörfler, M. Piwko, F. M. Wisser, T. Jaumann, E. Ahrens, L. Giebeler, H. Althues, S. Schädlich, J. Grothe, A. Jeffery, M. Grube, J. Brückner, J. Martin, J. Eckert, S. Kaskel, T. Mikolajick and W. M. Weber, Sci. Rep., 2016, 6, 27982.

10 M. Green and F.-M. Liu, A method of fabricating fibres composed of silicon or a silicon-based material and their use in lithium rechargeable batteries, WO 2007/083155 A1, 2007, pp. 1-14.

11 T. Jaumann, M. Herklotz, M. Klose, K. Pinkert, S. Oswald, J. Eckert and L. Giebeler, Chem. Mater., 2015, 27, 37-43.

12 X. H. Liu, L. Zhong, S. Huang, S. X. Mao, T. Zhu and J. Y. Huang, ACS Nano, 2012, 6, 1522-1531.

13 S. W. Lee, M. T. McDowell, J. W. Choi and Y. Cui, Nano Lett., 2011, 11, 3034-3039.

14 M. Green, E. Fielder, B. Scrosati, M. Wachtler and J. S. Moreno, Electrochem. Solid-State Lett., 2003, 6, A75-A79.
15 V. Pralong, D. C. S. Souza, K. T. Leung and L. F. Nazar, Electrochem. Commun., 2002, 4, 516-520.

16 R. Alcántara, J. L. Tirado, J. C. Jumas, L. Monconduit and J. Olivier-Fourcade, J. Power Sources, 2002, 109, 308-312.

17 Y. Kim, C. K. Lee, H. Sohn and T. Kang, J. Electrochem.Soc., 2004, 151, A933-A937.

18 B. Mauvernay, M. L. Doublet and L. Monconduit, J. Phys. Chem. Solids, 2006, 67, 1252-1257.

19 O. Crosnier and L. F. Nazar, Electrochem. Solid-State Lett., 2004, 7, A187-A189.

$20 \mathrm{~J}$. Wu and Z. Fu, J. Electrochem. Soc., 2009, 156, A22-A26.

21 J. Liu, P. Kopold, C. Wu, P. A. van Aken, J. Maier and Y. Yu, Energy Environ. Sci., 2015, 8, 3531-3538.

22 J. Qian, Y. Xiong, Y. Cao, X. Ai and H. Yang, Nano Lett., 2014, 14, 1865-1869.

23 J. Mao, X. Fan, C. Luo and C. Wang, ACS Appl. Mater. Interfaces, 2016, 8, 7147-7155.

24 W. Zhang, J. Mao, S. Li, Z. Chen and Z. Guo, J. Am. Chem. Soc., 2017, 139, 3316-3319.

25 W. Zhang, W. K. Pang, V. Sencadas and Z. Guo, Joule, 2018, 2, 1534-1547.

26 D. Duveau, S. Sananes Israel, J. Fullenwarth, F. Cunin and L. Monconduit, J. Mater. Chem. A, 2016, 4, 3228-3232.

27 H. Kwon, C. K. Lee, K. Jeon and C. Park, ACS Nano, 2016, 10, 5701-5709.

28 R. Reinhold, U. Stoeck, H.-J. Grafe, D. Mikhailova, T. Jaumann, S. Oswald, S. Kaskel and L. Giebeler, ACS Appl. Mater. Interfaces, 2018, 10, 7096-7106.

29 R. W. Olesinski, N. Kanani and G. J. Abbaschian, Bull. Alloy Phase Diagrams, 1985, 6, 130-133.

30 T. Wadsten, Acta Chem. Scand., 1969, 23, 2532-2560.

31 C. G. Beck and R. Stickler, J. Appl. Phys., 1966, 37, 4683-4687.

32 C. Barreteau, B. Michon, C. Besnard and E. Giannini, J. Cryst. Growth, 2016, 443, 75-80.

33 A.-Q. Cheng, Z. He, J. Zhao, H. Zeng and R.-S. Chen, ACS Appl. Mater. Interfaces, 2018, 10, 5133-5139.

34 B. Peng, Y. Xu, X. Wang, X. Shi and F. M. Mulder, Sci. China: Phys., Mech. Astron., 2017, 60, 064611.

35 M. T. McDowell, S. W. Lee, W. D. Nix and Y. Cui, Adv. Mater., 2013, 25, 4966-4985.

36 R. Glaum and R. Gruehn, Z. Anorg. Allg. Chem., 1989, 573, 2442.

37 F. Fauth, I. Peral, C. Popescu and M. Knapp, Powder Diffr., 2013, 28, S360-S370.

38 M. Herklotz, J. Weiß, E. Ahrens, M. Yavuz, L. Mereacre, N. Kiziltas-Yavuz, C. Dräger, H. Ehrenberg, J. Eckert, F. Fauth, L. Giebeler and M. Knapp, J. Appl. Crystallogr., 2016, 49, 340-345. 